\title{
Change-Point Models to Estimate the Limit of Detection
}

\author{
Ryan C. May ${ }^{a},{ }^{*}$, Haitao Chu ${ }^{b}$, Joseph G. Ibrahim ${ }^{c}$, Michael G. Hudgens ${ }^{c}$, Abigail C. Lees $^{d}$, \\ and David M. Margolis ${ }^{\mathrm{e}}$ \\ aThe EMMES Corporation, Rockville, Maryland 20850, U.S.A. \\ bDivision of Biostatistics, School of Public Health, University of Minnesota, Minneapolis, \\ Minnesota 55455, U.S.A. \\ 'Department of Biostatistics, University of North Carolina at Chapel Hill, Chapel Hill, North \\ Carolina 27599, U.S.A. \\ dDivision of Infectious Diseases, Department of Medicine, University of North Carolina at Chapel \\ Hill, Chapel Hill, North Carolina 27599, U.S.A. \\ eDepartment of Medicine, Epidemiology, Microbiology and Immunology, University of North \\ Carolina at Chapel Hill, Chapel Hill, North Carolina 27599, U.S.A.
}

\section{Abstract}

\begin{abstract}
In many biological and environmental studies, measured data is subject to a limit of detection. The limit of detection is generally defined as the lowest concentration of analyte that can be differentiated from a blank sample with some certainty. Data falling below the limit of detection is left-censored, falling below a level that is easily quantified by a measuring device. A great deal of interest lies in estimating the limit of detection for a particular measurement device. In this paper we propose a change-point model to estimate the limit of detection using data from an experiment with known analyte concentrations. Estimation of the limit of detection proceeds by a two-stage maximum likelihood method. Extensions are considered that allow for censored measurements and data from multiple experiments. A simulation study is conducted demonstrating that in some settings the change-point model provides less biased estimates of the limit of detection than conventional methods. The proposed method is then applied to data from an HIV pilot study.
\end{abstract}

\section{Keywords}

change point; limit of detection; linear calibration curve; two-stage maximum likelihood

\section{Introduction}

In many laboratory assays, interest resides in quantifying very dilute analyte concentrations in solution. At some low concentration level, a measured response cannot accurately be distinguished from background noise, the measured response from a blank sample. This low concentration point is called the limit of detection (LOD), a point that is specific to each particular measurement device [1]. Though the general definition given above for the limit of detection is widely accepted, the methodology used to determine the limit of detection is quite varied. In this paper we consider the estimation of the limit of detection using repeated measurements from known analyte concentrations. This analysis is motivated by the need to

Copyright (C) 2011 John Wiley \& Sons, Ltd.

*Correspondence to: Ryan May, The EMMES Corporation, 401 N Washington St, Rockville, Maryland 20850, U.S.A. rmay@emmes.com. 
determine the LOD of a novel assay that was developed to detect changes in low-level HIV expression after a drug intervention. In many assays, as concentrations decrease the resulting measured levels become more precise. In contrast, the assay measuring HIV expression becomes less precise as the concentration of HIV decreases. Additionally, operational restrictions related to the length of time required to perform the assay impose a limit on the measured HIV expression. As a result, measured HIV expression is censored at low levels, and assay measurements cannot be obtained from a blank sample. In this paper we consider estimating the LOD for this assay based on replicate measurements from several different known concentrations of HIV.

The rest of this article is organized as follows. In Section 2 we discuss past research on estimating the LOD, and develop the notation for the rest of the paper. We explain how previously considered models may mis-specify the error distribution for a measurement device, leading to biased estimates of the LOD. In Section 3, we introduce the proposed change-point model and discuss a two-stage estimation approach for obtaining maximum likelihood estimates of the LOD from the model. We also introduce a mixed model extension to the proposed approach to obtain global LOD estimates using data from multiple experiments. In Section 4, we examine the proposed change-point model using a simulation study. We apply the proposed model to the aforementioned HIV assay in Section 5. We conclude this article in Section 6 with a discussion.

\section{Background}

To distinguish between low analyte concentrations and those of a blank sample, many estimation approaches aim to quantify the distribution of measurements obtained from a blank sample. The distribution of assay measurements for a blank sample is often assumed to be Gaussian, with mean $\mu_{\text {blank }}$ and variance $\sigma_{\text {blank }}^{2}$ [2]. A limit of detection is then chosen to fall a "reasonable" distance outside of this blank distribution. Consequently, many definitions of LOD take the following form [3]:

$$
\mathrm{LOD}=\mu_{\text {blank }}+K \sigma_{\text {blank }}
$$

where $K$ is a definition-specific constant, usually in the range of 2 to 3 ([4], [5], [6]). When $K=3$, it is expected that $99.9 \%$ of measurements from a blank sample will fall below the limit of detection. Clearly, the larger value of $K$ that is chosen, the higher the LOD will be, and the lower the chance that a value from a blank will fall above the LOD. Using this definition, many estimation approaches are designed to accurately estimate $\mu_{\text {blank }}$ and $\sigma_{\text {blank }}$. In practice, such estimation is straightforward when many repeated measurements can be obtained from a blank sample, by taking the sample mean and standard deviation (SD) as estimates of $\mu_{\text {blank }}$ and $\sigma_{\text {blank }}$.

When blank measurements are not available, alternative estimation approaches can be utilized. One approach involves taking repeated measurements of a known low concentration of analyte and using these measurements as proxy measurements for a blank sample. In this case, the LOD definition is similar to (1). With $\mu_{\text {low }}$ and $\sigma_{\text {low }}$ representing the mean and standard deviation of the distribution of measurements at the low concentration (again assumed to follow a Gaussian distribution), the limit of detection is defined as:

$$
\mathrm{LOD}=\mu_{\text {low }}+K \sigma_{\text {low }}
$$

The previous definition of the LOD in (1) includes only a specification of the distribution of a blank sample. Using this definition enables direct control of the type I error, the chance of incorrectly specifying a blank sample as containing some concentration of analyte. For 
example, when $K=3$ the chance of a type I error is only $0.1 \%$ for any particular blank measurement. However, this specification does not control for type II error, the chance of incorrectly specifying a sample containing analyte as coming from a blank. If the type II error is high, clearly there is still difficulty in conclusively distinguishing a concentration value near the LOD from a blank. Consequently, many definitions of the LOD take both type I and type II error into account. To ensure that measured values for concentrations at the LOD are unlikely to fall in the range of a blank sample, alternative definitions of LOD account for the distribution of measurement values at some known small concentration of analyte. For example, the limit of detection is sometimes ([7], [4]) defined as:

$$
\mathrm{LOD}=\mu_{\text {blank }}+1.645 \sigma_{\text {blank }}+1.645 \sigma_{\text {low }}
$$

Under the assumption that measurements from a blank sample has a normal distribution with mean $\mu_{\text {blank }}$ and standard deviation $\sigma_{\text {blank }}, 95 \%$ of blank samples will fall below $\mu_{\text {blank }}+$ $1.645 \sigma_{\text {blank }}$ (called the "limit of blank", or "limit of decision"). Assuming that measurements from concentrations at the limit of detection defined by (3) have a normal distribution with mean equal to the LOD and standard deviation $\sigma_{\text {low }}, 95 \%$ of concentration measurements from concentrations at the limit of detection will fall above the limit of blank. It should be noted that definition (3) is only needed when it is assumed that the measurement standard deviation for a blank sample is different from the standard deviation for any other "low" concentration sample at or around the LOD (i.e. $\sigma_{\text {blank }} \neq \sigma_{\text {low }}$ ). Many authors ([2], [8], [4]) assume a constant measurement error variance for any true concentrations near or below the limit of detection. In this case, the choice of $K$ in (1) specifies the chance of a type I or type II error. When $K=3.29$, the chance of either type of misclassification is $5 \%$; when $K=$ 3 , the chance is $7 \%$. Alternative (but similar) definitions to (3) calculate a pooled measurement standard deviation from both blank and low samples, using the pooled estimate in place of both $\sigma_{\text {blank }}$ and $\sigma_{\text {low }}$ [5].

The LOD definitions displayed in equations (1) and (3) usually are employed under the assumption that the measurement distribution for a blank sample is Gaussian. Estimation of the LOD using a Gaussian assumption can be more efficient than nonparametric methods if the Gaussian assumption approximates the underlying distribution of a blank sample. In reality, characteristics of the measurement device often result in non-Gaussian measurement distributions for a blank. In such cases, nonparametric methods have been proposed [9], which involve estimating quantiles from the observed blank distribution. For definition (3), a nonparametric estimate of the 95th quantile can be used in place of $\mu_{\text {blank }}+1.645 \sigma_{\text {blank }}$. In this case, the value $\sigma_{\text {low }}$ can still be estimated with parametric methods when it is reasonable to assume a Gaussian distribution for a low concentration sample. If the low concentration cannot be assumed Gaussian, nonparametric estimation of the 5th and 50th quantiles of the low concentration sample can be used to complete equation (3). In practice, the limited number of replicates obtained make nonparametric estimation of quantiles less efficient than estimation that relies on parametric assumptions. Consequently, most analyses rely on the Gaussian distribution assumption, and define the limit of detection as in (3).

In practice, data analysts often do not have access to replicates of data from blank or "low" concentrations. This is the case for the HIV pilot study data considered in Section 5, in which the number of polymerase chain reaction (PCR) cycles needed to obtain a blank sample measurement is too high to be operationally feasible. In this case it is difficult to directly estimate the distribution of measurements for a blank sample, or the distribution of any low concentration sample. In such settings estimation often proceeds using higher analyte concentrations from which measurements are more easily obtained. A regression line is then fit to $\left(X_{1}, Y_{1}\right), \ldots,\left(X_{n}, Y_{n}\right)$, the $n$ observed pairs of analyte concentrations $X$ and 
measured responses $Y$. This fitted regression line is known as a linear calibration curve. Assuming a linear relationship between $X$ and $Y$, we have the following model specification ([10], [11]):

$$
Y_{i}=\beta_{0}+\beta_{1} X_{i}+\epsilon_{i}, \quad \epsilon_{i} \sim N\left(0, \sigma^{2}\right)
$$

The assumptions of (4) specify the distribution of $Y_{i} \mid X_{i} \sim N\left(\beta_{0}+\beta_{1} X_{i}, \sigma^{2}\right)$. Clearly, the parameter estimates of the model can be used to directly estimate the distribution of $Y \mid X_{i}=0$, the response for a blank sample. We have:

$$
Y \mid X_{i}=0 \sim N\left(\beta_{0}, \sigma^{2}\right)
$$

Using the definition of LOD in equation (1) with $K=3$, the LOD under model (4) is:

$$
\mathrm{LOD}_{Y}=\beta_{0}+3 \sigma
$$

The above specification is conditional on the true values of the model parameters $\beta_{0}, \beta_{1}$, and $\sigma^{2}$. Let $\widehat{\beta}_{0}, \widehat{\beta}_{1}$ and $\widehat{\sigma}^{2}$ denote the maximum likelihood estimates (MLE's) for $\beta_{0}, \beta_{1}$, and $\sigma^{2}$ (and denote the estimated variance of $\widehat{\beta}_{0}$ by $\widehat{\sigma}_{\beta_{0}}^{2}$ ). The response distribution for a blank sample can be estimated as follows:

$$
Y \mid X_{i}=0 \sim N\left(\widehat{\beta}_{0}, \widehat{\sigma}^{2}+\widehat{\sigma}_{\beta_{0}}^{2}\right)
$$

Consequently, the limit of detection can be estimated as ([12], [11]):

$$
\widehat{\mathrm{LOD}}_{Y}=\widehat{\beta}_{0}+3\left(\widehat{\sigma}^{2}+\widehat{\sigma}_{\beta_{0}}^{2}\right)^{1 / 2}
$$

In practice, the limit of detection is usually defined in terms of the concentration $X$ instead of the measurement $Y$. To obtain the limit of detection for concentration, a simple linear transformation on $\widehat{\mathrm{LOD}}_{Y}$ is performed [13], to obtain:

$$
\widehat{\operatorname{LOD}}_{X}=\frac{3\left(\widehat{\sigma}^{2}+\widehat{\sigma}_{\beta_{0}}^{2}\right)^{1 / 2}}{\widehat{\beta}_{1}}
$$

The standard analysis for estimating the LOD with a linear calibration curve assumes that the variance of measured responses is constant at all concentration values. In many practical applications this is not the case, and it is common for a measurement device to become more (or less) precise as the concentration of analyte increases. In this case, it may be more appropriate to allow the measurement standard deviation (or variance) to change with analyte concentration. In the most basic case (or possibly under suitable transformation), the measurement standard deviation is assumed to change linearly with the concentration as follows: 


$$
Y_{i}=\beta_{0}+\beta_{1} X_{i}+\epsilon_{i}, \quad \epsilon_{i} \sim N\left(0,\left(\sigma_{0}+\sigma_{1} X_{i}\right)^{2}\right)
$$

Using this specification, the limits of detection $L O D_{X}$ and $L O D_{Y}$ are again estimated as in equations (5) and (6).

The previously discussed methods for estimating the limit of detection with a linear calibration curve either assume a constant standard deviation for measurement error as in (4), or a change in measurement standard deviation by concentration as in (7). As noted by several authors ([8], [11], [1], [4]), a more realistic assumption may be that the measurement standard deviation changes for sufficiently high concentration values above the limit of detection, while remaining effectively constant for sufficiently low concentration values. Under this assumption, the use of a constant standard deviation model like (4) for all concentration values may result in underestimation (if precision increases with concentration) or overestimation (if precision decreases with concentration) of the limit of detection. The use of a linear standard deviation model like (7) could provide the opposite effect, overestimating the LOD when precision increases with concentration and underestimating when precision decreases with concentration. One solution to this issue was proposed by Rocke and Lorenzato [14], who partitioned measurement error terms into proportional and additive terms. Taking $\eta_{i}$ and $\varepsilon_{i}$ as the component error terms, the Rocke and Lorenzato model is written as:

$$
Y_{i}=\beta_{0}+\beta_{1} X_{i} e^{\eta_{i}}+\epsilon_{i} \quad \eta_{i} \sim N\left(0, \sigma_{\eta}^{2}\right), \quad \epsilon_{i} \sim N\left(0, \sigma_{\epsilon}^{2}\right)
$$

For low concentrations (with $X_{i}$ close to zero), the measurement error is approximately constant as in (4). For larger concentration values, the measurement error is proportional to the true concentration as in (7). While this approach provides a more flexible framework for the error distribution than either the constant standard deviation model (4) or the linear standard deviation model (7), the measurement error distribution is not specified as constant for low concentrations below the LOD. If in fact the error standard deviation is constant below the LOD, assuming the Rocke-Lorenzato model could yield biased LOD estimates. In the next section a change-point model is proposed that includes models (4) and (7) as special cases and allows for the possibility that the error standard deviation becomes constant at concentration levels below the LOD.

\section{Proposed Change-Point Models}

\subsection{Regression Model}

Because measurements below the LOD are indistinguishable from a blank, it follows that the measurement standard deviation may be constant for low analyte concentrations. Such a distribution can be modeled using a change-point for the measurement standard deviation. While the literature on change-point models in both regression ([15], [16]) and mixed [17] modeling is quite rich, to our knowledge no published articles have looked at models with a change-point on the standard deviation of the error. Here we consider the model:

$$
Y_{i}=\beta_{0}+\beta_{1} X_{i}+\epsilon_{i}, \quad \epsilon_{i} \sim N\left(0, \sigma_{i}^{2}\right)
$$

where 


$$
\sigma_{i}=\left\{\begin{array}{lll}
\sigma_{0} & \text { if } & X_{i} \leq \lambda \\
\sigma_{0}+\sigma_{1}\left(X_{i}-\lambda\right) & \text { if } & X_{i}>\lambda
\end{array}\right.
$$

Here, $\lambda$ represents the change-point for measurement standard deviation. As noted in Section 2 , a common definition for the LOD is three standard deviations away from the expected value of a blank sample. Adopting this convention, under model (9) the standard deviation and expected value of a blank sample are $\sigma_{0}$ and $\beta_{0}$, such that the LODs for the measurement $(\mathrm{Y})$ and true concentration $(\mathrm{X})$ are:

$$
\begin{gathered}
\operatorname{LOD}_{Y}=\beta_{0}+3 \sigma_{0} \\
\operatorname{LOD}_{X}=\frac{3 \sigma_{0}}{\beta_{1}}
\end{gathered}
$$

Taking $\widehat{\beta}_{0}, \widehat{\sigma}_{0}, \widehat{\sigma}_{\beta 0}$, and $\widehat{\beta}_{1}$ as the MLE's for their respective parameters, the limit of detection is estimated following equations (5) and (6) with $\widehat{\sigma}^{2}$ replaced by $\widehat{\sigma}_{0}^{2}$. In the HIV pilot study motivating this paper the measured assay responses $Y$ are right-censored at a constant upper limit (here denoted as $\gamma$ ). Accounting for this censoring, the log-likelihood for an individual observation is:

$$
l_{i}\left(\beta_{0}, \beta_{1}, \sigma_{0}, \sigma_{1}, \lambda\right)= \begin{cases}-\log \left(\sigma_{i}\right)-\frac{1}{2 \sigma_{i}^{2}}\left\{Y_{i}-\left(\beta_{0}+\beta_{1} X_{i}\right)\right\}^{2} & \text { if } \quad Y_{i} \leq \gamma \\ \log \left[1-\Phi\left\{\frac{\gamma-\left(\beta_{0}+\beta_{1} X_{i}\right)}{\sigma_{i}}\right\}\right] & \text { if } \quad Y_{i}>\gamma\end{cases}
$$

where $\Phi()$ is the cumulative distribution function of a standard normal random variable. Therefore the log-likelihood for the model can be expressed as:

$$
l\left(\beta_{0}, \beta_{1}, \sigma_{0}, \sigma_{1}, \lambda\right)=\sum_{i=1}^{n} l_{i}\left(\beta_{0}, \beta_{1}, \sigma_{0}, \sigma_{1}, \lambda\right)
$$

In order to estimate the LOD under model (9), we maximize the log-likelihood (12) with respect to the parameter vector $\left(\beta_{0}, \beta_{1}, \sigma_{0}, \sigma_{1}, \lambda\right)$. Maximization of the log-likelihood is done under the following constraints. First, the change-point $(\lambda)$ must be constrained within the range of the observed $X_{i}$. Taking $x_{(1)}, \ldots, x_{(n)}$ as the order statistics for the observed $X_{i}$, this is expressed as $x_{(1)} \leq \lambda \leq x_{(n)}$. The rationale for this constraint is that the parameters $\sigma_{0}$ and $\lambda$ become unidentifiable when $\lambda \leq x_{(1)}$, and the parameters $\sigma_{1}$ and $\lambda$ become unidentifiable when $\lambda \geq x_{(n)}$.

The second model constraint is that the error standard deviation $\sigma_{i}$ cannot be negative at $x_{(1)}$, and the third model constraint is that $\sigma_{i}$ cannot be negative at $x_{(n)}$. Together, the second and third constraints specify that $\sigma_{i}$ is nonnegative at all points in $\left[x_{(1)}, x_{(n)}\right]$. These constraints are satisfied by requiring $\sigma_{0} \geq 0$ and $\sigma_{0}+\sigma_{1}\left(x_{(n)}-\lambda\right) 0$. All constraints on the model are given below:
i. $\quad x_{(1)} \leq \lambda \leq x_{(n)}$
ii. $\quad \sigma_{0} \geq 0$ 
iii. $\sigma_{0}+\sigma_{1}\left(x_{(n)}-\lambda\right) \geq 0$

Constraints (i) and (ii) are both linear, so are straightforward to implement when maximizing (12). However, constraint (iii) is not linear, as it involves the term $\sigma_{1} \lambda$. Therefore, maximizing (12) subject to (i), (ii), and (iii) is challenging since many standard optimization routines only allow for linear constraints. To get around this issue, we instead use a two-stage optimization routine [18]. For ease of exposition, define $\sigma_{x(n)}=\sigma_{0}+\sigma_{1}\left(x_{(n)}\right.$ $-\lambda)$, the standard deviation at $x_{(n)}$, the maximum observed concentration value. For generic parameter $\varphi$, we denote $\varphi^{(t)}$ as the parameter estimate at the $t$-th iteration of the estimation routine. The proposed two-stage optimization routine is as follows:

1. Fix $\lambda=\lambda^{(t-1)}$. Maximize (12) with fixed $\lambda$, subject to the linear constraints:

i. $\quad \sigma_{0} \geq 0$

ii. $\quad \sigma_{0}+\sigma_{1}\left(x_{(n)}-\lambda\right) \geq 0$

2. Taking $\widehat{\sigma}_{0}$ and $\widehat{\sigma}_{1}$ as the estimates from step 1 , fix $\sigma_{x_{(n)}}=\widehat{\sigma}_{0}+\widehat{\sigma}_{1}\left(x_{(n)}-\lambda^{(t-1)}\right)$. Maximize (12) with fixed $\sigma_{x_{(n)}}$ subject to the linear constraints:

$$
\text { i. } x_{(1)} \leq \lambda \leq x_{(n)}
$$

ii. $\quad \sigma_{0} \geq 0$

Obtain estimates $\beta_{0}^{(t)}, \beta_{1}^{(t)}, \sigma_{0}^{(t)}, \lambda^{(t)}$, set $\sigma_{1}^{(t)}=\left(\sigma_{x_{(n)}}-\sigma_{0}^{(t)}\right) /\left(x_{(n)}-\lambda^{(t)}\right)$

Steps 1 and 2 in the above procedure are repeated until convergence is achieved for all parameter estimates. The convergence criterion used for parameter $\varphi$ specifies that $\mid \varphi^{(t)}$ $\varphi^{(t-1)} \mid \leq k$, with $k$ representing a generic constant. The proposed optimization routine is relatively simple to implement, as the likelihood in (12) is not overly complex. For model (9) analysis results presented in Sections 4 and 5, optimization in each stage was performed using R software [19] with the constrOptim() function.

Standard large sample approximations of the distribution of the MLE are in general not justified in this setting as the change-point parameter is not regular [20]. Alternatively, various forms of the bootstrap have been shown to be theoretically justified and to perform well empirically in change-point problems. Therefore, in the HIV example presented in Section 5 the bootstrap is employed to estimate the standard error of the LOD estimate.

\subsection{Mixed Model}

The regression model (9) can be used to estimate the limit of detection for any individual experiment. Extending this approach, a linear mixed effects model was also considered to allow simultaneous estimation of a single limit of detection from multiple experiments. The model specification is given as follows:

$$
\begin{aligned}
& Y_{i j}=\beta_{0}+\beta_{1} X_{i j}+b_{i 0}+b_{i 1} X_{i j}+\epsilon_{i j}, \quad \epsilon_{i j} \sim N\left(0, \sigma_{i j}^{2}\right) \\
& \sigma_{i j}=\left\{\begin{array}{lll}
\sigma_{0} & \text { if } & X_{i j} \leq \lambda_{i} \\
\sigma_{0}+\sigma_{1}\left(X_{i j}-\lambda_{i}\right) & \text { if } & X_{i j}>\lambda_{i}
\end{array}\right. \\
& \left(b_{i 0}, b_{i 1}\right) \sim B V N\left(\mathbf{0},\left[\begin{array}{cc}
\sigma_{b_{0}}^{2} & \rho_{b_{0}} \sigma_{b_{1}} \\
\rho \sigma_{b_{0}} \sigma_{b_{1}} & \sigma_{b_{1}}^{2}
\end{array}\right]\right)
\end{aligned}
$$

where $Y_{i j}$ is the measured assay response and $X_{i j}$ is the true analyte concentration for experiment $i$ and measurement $j$, and $B V N$ denotes a bivariate normal distribution. The parameters $\beta_{0}$ and $\beta_{1}$ represent the main effect parameters, with $b_{i 0}$ and $b_{i 1}$ representing the 
experiment-specific random effects. The values of $\sigma_{0}$ and $\sigma_{1}$ are not experiment-specific, to allow estimation of a population-averaged limit of detection. The change-point $\lambda_{i}$ is experiment-specific. In the presence of right-censored data, model (13) can be modified in a manner analogous to the approach used for model (9). Maximum likelihood estimation for this model was performed using PROC NLMIXED in SAS software version 9.3. Unlike the PROC MIXED procedure (which is more commonly used to fit linear mixed effects models), the NLMIXED procedure can account for right-censored data and the changepoint standard deviation in model (13). In Section 5, this model is applied to data from an HIV pilot study.

\section{Simulation Study}

A simulation study was conducted to analyze the performance of the proposed change-point model. The data for this simulation study was generated using model (9), with a parameter specification that mirrored the HIV data analysis presented in Section 5. Only five different values of concentration $X_{i}$ were used at 1,2,3,4, and 5. For each of the concentration values, repeated measurements $\left(Y_{i}\right)$ were generated. The number of $Y_{i}$ generated for each of the five concentration values was equal, a balanced allocation. For all simulations, the parameter values were specified as follows: $\beta_{0}=45, \beta_{1}=-3.7, \sigma_{0}=1$.1. Four different sets of simulations were run using a different value for the change-point (denoted as Case 1, 2, 3, and 4 ), with $\lambda$ taking values $1.5,2.5,3.5$, and 4.5. The value of $\sigma_{X(n)}$, the standard deviation at the maximum concentration value, was kept constant at 0.25 for all simulations (again mirroring results from the HIV data analysis). The specified values of $\sigma_{0}, \lambda$, and $\sigma_{X(n)}$ determined the parameter value of $\sigma_{1}$ for each simulated data set. Following the data set in Section 5, all values of $Y_{i}$ falling above 42 were set as right-censored. For comparison, data was simulated from two additional specifications for $\sigma_{i}$ not involving a change-point. The first (Case 5) used a constant value for $\sigma_{i}$, following the specification given by (4). The second (Case 6) used the linear specification for $\sigma_{i}$ given by (7).

For each simulation scenario, 10,000 data sets of size $n=80,150$, and 300 were generated. The proposed change-point model was then fit to the data, using the two-stage estimation approach described in Section 3.1 to obtain maximum likelihood estimates of all the model parameters. For comparison, model (7) assuming a linear change in standard deviation with no change-point and model (4) assuming constant standard deviation were also fit to the simulated data sets. Both comparison models were modified slightly to account for censoring of the response variable, similar to the method for the change point model presented in Section 3.1.

Table 1 presents the empirical bias and standard deviation (SD) of the 10,000 estimates for selected parameters in each model. Under the change-point specification for $\sigma_{i}$ in Cases 1-4, the change-point model exhibited less bias in estimating the $L O D_{X}$ than both the linear standard deviation and constant standard deviation models, for every sample size considered. The change-point model tended to slightly underestimate the limit of detection, particularly when the change-point was small relative to the range of the observed concentration values. As expected, the constant standard deviation model (4) provided a poor estimate of the $L O D_{X}$, with a negative bias for all cases considered. The magnitude of this bias tended to increase when the change-point was low relative to the range of observed data. The linear standard deviation model (7) also provided a poor estimate of the $L O D_{X}$, by overestimating the $L O D_{X}$ for all cases considered. This bias increased when the changepoint was high relative to the range of observed data. The change-point model also produced $L O D_{X}$ estimates with a smaller standard deviation than the linear standard deviation model for all sample sizes considered. Similar relationships were seen for the estimates of $\sigma_{0}$ and $\sigma_{1}$, with the change-point model generally providing estimates with the least bias, and 
estimates with smaller standard deviation than the linear model. Increased sample size did not seem to affect the bias in any of the models considered, though the standard deviations of the $L O D_{X}$ estimates decreased.

Under alternative specifications for $\sigma_{i}$ not involving a change-point (Cases 5 and 6), the benefits of the change-point model were less clear. In Case 5, with a constant specification for $\sigma_{i}$, the constant standard deviation model understandably provided estimates with the least bias. Correspondingly, the linear standard deviation model provided estimates with least bias in Case 6, which used a linear specification for $\sigma_{i}$. However, in neither case did the change-point model give the most biased estimates, providing less bias than the linear model in Case 5 and less bias than the constant model in Case 6. The precision of estimates followed the trend seen in Cases 1-4, with the constant model providing the most precise estimates and the linear model providing the least precise estimates.

In addition to parameter estimates, the Akaike Information Criterion (AIC, [21]) was also calculated for each of the three models fit to every simulated data set. For each set of 10,000 simulated data sets, the AIC value for the change-point, constant, and linear models was calculated. Of these three models, the one with the lowest AIC was selected as the best fit for the current data set. Table 1 displays the proportion of the 10,000 simulated data sets that resulted in a particular model having the best fit. For example, in Case 4 with $n=80$, the change-point model had the best model fit in $96.0 \%$ of the simulated data sets, compared to $0.6 \%$ for the linear standard deviation model and $3.4 \%$ for the constant standard deviation model. The results displayed in Table 1 show that under the change-point specification in Cases 1-4, the change-point model produces the best fit to the data a much higher proportion of the time than either the linear standard deviation or constant standard deviation models. This "relative fit" of the change-point model tended to increase with increasing change point, and also with sample size, from $60.3 \%$ in the $n=80, \lambda=1.5$ simulation (Case 1) to $100 \%$ in the $n=300, \lambda=4.5$ simulation (Case 4). Results were more mixed under the alternative specifications (Cases 5 and 6). Under a constant standard deviation specification in Case 5, the change-point model provided the best fit only $3 \%$ of the time when $n=80$, and only $1 \%$ of the time when $n=300$. Under the linear standard deviation specification in Case 6 , the change-point model provided the best fit only $18 \%$ of the time when $n=80$, but $56 \%$ of the time when $n=300$.

\section{HIV Data}

Data for this analysis comes from a pilot study analyzing the effects of a drug on HIV transcription. Resting cells from HIV infected patients were treated with the drug, with interest in the degree to which HIV transcription was increased. HIV RNA in general is too unstable and must be reverse transcribed into the more stable form, cDNA. The concentration of HIV RNA in patient samples was much too low to be directly measured, and following conversion to cDNA subsequent amplification by real-time PCR was necessary ([22], [23]). The region of the HIV genome that was amplified in this assay codes for a highly conservative region known as gag which was measured with primers and probes as described by Agarwal et al. [24]. RNA from patient samples was quantified using a linear calibration curve based on known concentrations of HIV cDNA. The PCR machine measures unknown quantities through fluorescence that is proportional to sample concentration and amplifies over many cycles. A cycle-threshold is defined as the PCR cycle that results in the highest increase in fluorescence. By comparing the cycle-threshold value for a given unknown concentration of RNA to a linear calibration curve for different known HIV concentrations, the unknown concentration was estimated. 
For each patient in the pilot study, a linear calibration curve was created by measuring the cycle-threshold value for different known concentrations of HIV. Data for the study consists of calibration curve data for six experiments (one for each patient, six patients total), with each experiment consisting of 20 measurements for each of four or five known concentrations of HIV. The goal of this analysis was to estimate the LOD for the concentration of HIV individually for each experiment. Complicating the analysis is the restriction that each sample was run for a maximum of 42 cycles of PCR amplification; HIV concentrations resulting in more than 42 cycles were right-censored.

It is important to note here that the concentration of HIV $(X)$ is inversely related to the cyclethreshold value $(Y)$ in the analyzed data. A lower concentration of HIV in general takes more PCR cycles to fluoresce, resulting in a higher cycle-threshold value. This relationship is the opposite of what is usually observed when relating known concentrations to measured values, where measurement $(Y)$ usually increases with analyte concentration $(X)$. Because of the inverse relationship between $Y$ and $X$ in the current data, the $L O D$ estimates will be slightly altered from (5) and (6), taking the form:

$$
\begin{aligned}
& \widehat{\mathrm{LOD}}_{Y}=\widehat{\beta}_{0}-3\left(\widehat{\sigma}_{0}^{2}+\widehat{\sigma}_{\beta_{0}}^{2}\right)^{1 / 2} \\
& \widehat{\mathrm{LOD}}_{X}=\frac{-3\left(\widehat{\sigma}_{0}^{2}+\widehat{\sigma}_{\beta_{0}}^{2}\right)^{1 / 2}}{\widehat{\beta}_{1}}
\end{aligned}
$$

Analysis of the data was performed in two ways. First, the change-point model proposed in Section 3.1 was fit separately for each individual experiment, generating experimentspecific LOD estimates. As with the simulation study, both linear standard deviation and constant standard deviation models were included for comparison. The model fit was again analyzed using the AIC. In addition, the mixed model proposed in Section 3.2 was fit to data from all six experiments simultaneously, to generate a population-averaged estimate of the LOD.

Parameter estimates for both the regression and mixed model approaches are given in Table 2, and a plot of the model fit for experiments 1 and 3 is given in Figure 1. The dashed lines about the predicted regression line in Figure 1 represent $95 \%$ prediction intervals for the data, with the vertical and horizontal dashed lines representing the estimated LOD.

Estimates of experiment-specific LODs (denoted $\widehat{L O D}_{X}$ in Table 2) using the change-point model range from 0.468 to 1.195 , which correspond to LOD estimates on the untransformed HIV concentrations of 2.94 to 15.68 copies of gag. LOD estimates from the change-point model were lower than those from the linear standard deviation model, and were higher than estimates from the constant standard deviation model, for all experiments. The AIC for the change-point model was lower than the AIC for the linear standard deviation model in only one of the six experiments tested, suggesting that the linear standard deviation model generally provided a better fit to the data when the regression model was utilized. In experiments $1,2,5$, and 6 , the change-point estimates equal 1.0, the lowest observed concentration value. This makes the likelihood for the model identical to the linear standard deviation model (notice the identical parameter estimates for $\beta_{0}$ and $\beta_{1}$ ), only with more parameters estimated in the change-point model. This results in the higher AIC value for the change-point model.

The mixed model results also give LOD estimates for the change-point model that are higher than the constant standard deviation model, and lower than the linear standard deviation model. The untransformed LOD estimate of 15.49 is in the range of LOD estimates for the regression change-point models on each experiment, as expected. The AIC results indicate 
that the change-point model provides a better fit to the available data than does the linear standard deviation or constant standard deviation mixed models.

\section{Discussion}

In this paper a change-point model is proposed to estimate the limit of detection with a linear calibration curve. In certain settings, the proposed approach may provide a more realistic model of the underlying distribution of measurement errors in a linear calibration curve. Estimation is performed via a two-stage estimation technique, such that the nonlinear constraints on the model parameters are satisfied. Application of the proposed model was demonstrated using both an individual regression model and a mixed model.

The simulation results presented in Table 1 demonstrate that the proposed change-point model can improve estimation of the limit of detection when compared to both the linear standard deviation and constant standard deviation models. When measurement error is constant for low concentrations of analyte, the linear standard deviation model tends to overestimate the measurement error for a blank sample, and consequently tends to overestimate the limit of detection. This is shown in Table 1, where estimates using the change-point model exhibit smaller bias than the linear standard deviation model, particularly when some of the observed concentrations fall below the true change-point. The constant standard deviation model was shown to underestimate the LOD for all simulations considered, with a substantially larger bias than the change-point model. When AIC fit statistics were analyzed, the change-point model was correctly identified as the model providing the best fit for all cases where the data were simulated under this model.

The key assumption of the proposed change-point model is that the measurement error standard deviation is constant below some low concentration value. If this assumption does not hold (the standard deviation instead continues to increase or decrease with concentration), the change-point model would be expected to exhibit a greater bias than the linear standard deviation model. In this case, when the measurement error increases with concentration, the change-point model would tend to overestimate the limit of detection. When the measurement error decreases with concentration, the change-point model would tend to underestimate the LOD.

The proposed change-point model specifies a linear relationship between analyte concentration and measurement standard deviation for concentrations above the changepoint. Often in the analysis of linear calibration curves it is common to instead specify the error distribution in terms of the measurement variance. This approach was first considered by Oppenheimer et al. [25], who modeled the error variance $\sigma_{i}^{2}$ as a function of concentration $X_{i}$ via $\sigma_{i}^{2}=\sigma_{0}^{2}+\sigma_{1}^{2} X_{i}+\sigma_{2}^{2} X_{i}^{2}$. Such an approach could easily be extended to incorporate a change-point if it was thought that changing measurement error could more accurately be modeled in terms of the variance. Our choice to model the measurement standard deviation instead of variance was motivated by an inspection of measurement error in the available HIV data.

The proposed linear regression change-point model is quite straightforward to implement, and convergence of the parameter estimates was achieved very quickly in both the simulation and HIV analyses. The mixed model approach in Section 3.2 also converged very quickly, making the proposed approaches quite feasible. However, the profile likelihood plots for the change-point parameter $\lambda$ (not shown) indicate that the likelihood surface may in some instances not be very well-behaved, and that estimation by iterative procedures may not always be straightforward. The plots also reinforce the importance of using the bootstrap 
to estimate standard errors. Further theoretical investigation may shed some additional light on this potential issue.

\section{Acknowledgments}

The authors thank three anonymous reviewers for helpful comments that substantially improved the paper.

The work was partially supported by the UNC Center for AIDS Research NIH grant P30A1050410 (to Dr. Chu and Dr. Hudgens), the International Network for Strategic Initiatives in Global HIV Trial, NIH/NIAID grant U01AI068641 (to Dr. Chu), and by NIH grant R01 AI029168 (to Dr. Hudgens).

Contract/grant sponsor: NIH; contract/grant numbers: P30A1050410

\section{References}

1. Clinical and Laboratory Standards Institute. Protocols for Determination of Limits of Detection and Limits of Quantitation, Approved Guideline. CLSI document EP17 2004. CLSI; Wayne, PA USA:

2. Anderson DJ. Determination of the Lower Limit of Detection [letter]. Clinical Chemistry. 1989; 35:2152-3. [PubMed: 2619804]

3. Whitcomb BW, Schisterman EF. Assays with Lower Detection Limits: Implications for Epidemiological Investigations. Paediatric and Perinatal Epidemiology. 2008; 22:597-602. [PubMed: 19000298]

4. Browne RW, Whitcomb BW. Procedures for Determination of Detection Limits. Epidemiology. 2010; 21(4):S4-S9. [PubMed: 20220523]

5. Long GL, Winefordner JD. Limit of Detection: a Closer Look at the IUPAC Definition. Analytical Chemistry. 1983; 55:712A-724A.

6. Thomsen V, Schatzlein D, Mercuro D. Limits of Detection in Spectroscopy. Spectroscopy. 2003; 18(12):112-114.

7. Armbruster DA, Pry T. Limit of Blank, Limit of Detection and Limit of Quantitation. The Clinical Biochemist Reviews. 2008; 29:S49-S52.

8. Armbruster DA, Tillman MD, Hubbs LM. Limit of Detection (LOD)/Limit of Quantitation (LOQ): Comparison of the Empirical and the Statistical Methods Exemplified with GC-MS Assays of Abused Drugs. Clinical Chemistry. 1994; 40(7):1233-1238. [PubMed: 8013092]

9. Linnet K, Kondratovich M. Partly Nonparametric Approach for Determining the Limit of Detection. Clinical Chemistry. 2004; 50(4):732-740. [PubMed: 14764644]

10. Dunne A. Decision and Detection Limits for Linear Homoscedastic Assays. Statistics in Medicine. 1995; 14:1949-1959. [PubMed: 8677396]

11. Hubaux A, Vos G. Decision and Detection Limits for Linear Calibration Curves. Analytical Chemistry. 1967; 42(8):849-855.

12. Cox C. Limits of Quantitation for Laboratory Assays. Applied Statistics. 2005; 54:63-76.

13. Gibbons RD, Grams NE, Jarke FH, Stoub KP. Practical Quantitation Limits. Chemometrics and Intelligent Laboratory Systems. 1992; 12:225-235.

14. Rocke DM, Lorenzato S. A Two-Component Model for Measurement Error in Analytical Chemistry. Technometrics. 1995; 37(2):176-184.

15. Bai J. Estimation of a Change Point in Multiple Regression Models. Review of Economics and Statistics. 1997; 79(4):551-563.

16. Hawkins DM. Fitting Multiple Change-Point Models to Data. Computational Statistics \& Data Analysis. 2001; 37:323-341.

17. Cudeck R, Klebe K. Multiphase Mixed-Effects Models for Repeated Measures Data. Psychological Methods. 2002; 7(1):41-63. [PubMed: 11928890]

18. Smyth GK. Partitioned Algorithms for Maximum Likelihood and Other Non-Linear Estimation. Statistics and Computing. 1996; 6:201-216. 
19. R Development Core Team. R: A Language and Environment for Statistical Computing. R Foundation for Statistical Computing; Vienna, Austria: 2008. ISBN 3-900051-07-0, URL http:// www.R-project.org

20. Pawitan, Y. Encyclopedia of Biostatistics. John Wiley and Sons, Ltd; 2005. Change-point Problem..

21. Akaike H. A New Look at the Statistical Model Identification. IEEE Transactions on Automatic Control. 1974; 19(6):716-723.

22. Nolan T, Hands RE, Bustin SA. Quantification of mRNA Using Real-Time RT-PCR. Nature Protocols. 2006; 1(3):1559-1582.

23. Palmer S, Wiegand AP, Maldarelli F, Bazmi H, Mican JM, Polis M, Dewar RL, Planta A, Liu SY, Metcalf JA, Mellors JW, Coffin JM. New Real-Time Reverse Transcriptase-Initiated PCR Assay with Single-Copy Sensitivity for Human Immunodeficiency Virus Type 1 RNA in Plasma. Journal of Clinical Microbiology. 2003; 41(10):4531-4536. [PubMed: 14532178]

24. Agarwal A, Sankaran S, Vajpayee M, Sreenivas V, Seth P, Dandekar S. Correlation of Immune Activation With HIV-1 RNA Levels Assayed by Real-Time RT-PCR in HIV-1 Subtype C Infected Patients in Northern India. Journal of Clinical Virology. 2007; 40(4):301-306. [PubMed: 17962068]

25. Oppenheimer L, Capizzi TP, Weppelman RM, Mehta H. Determining the Lowest Limit of Reliable Assay Measurement. Analytical Chemistry. 1983; 55:638-643. 


\section{Experiment 1 Results}

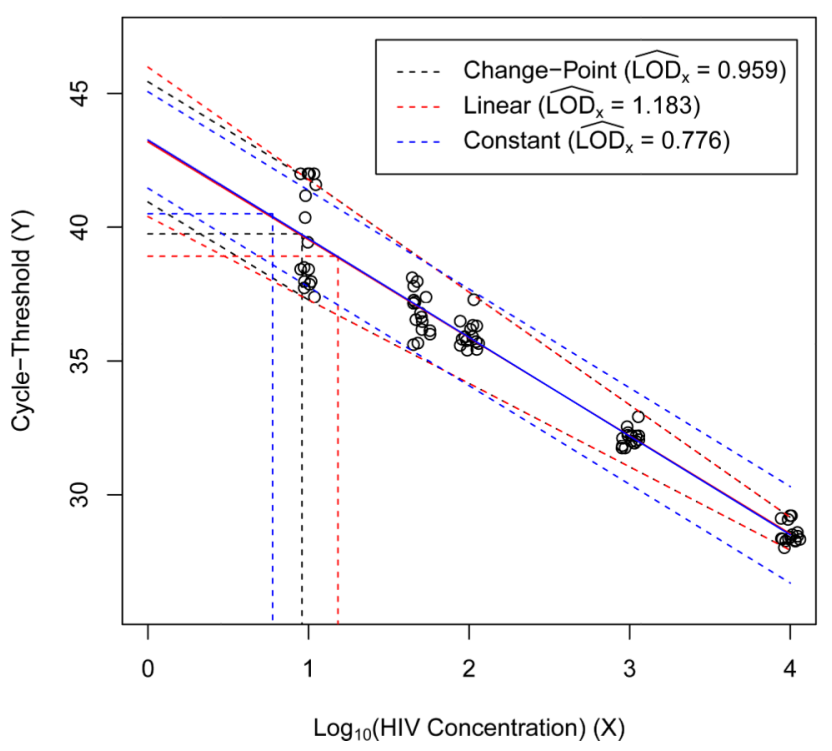

Experiment 3 Results

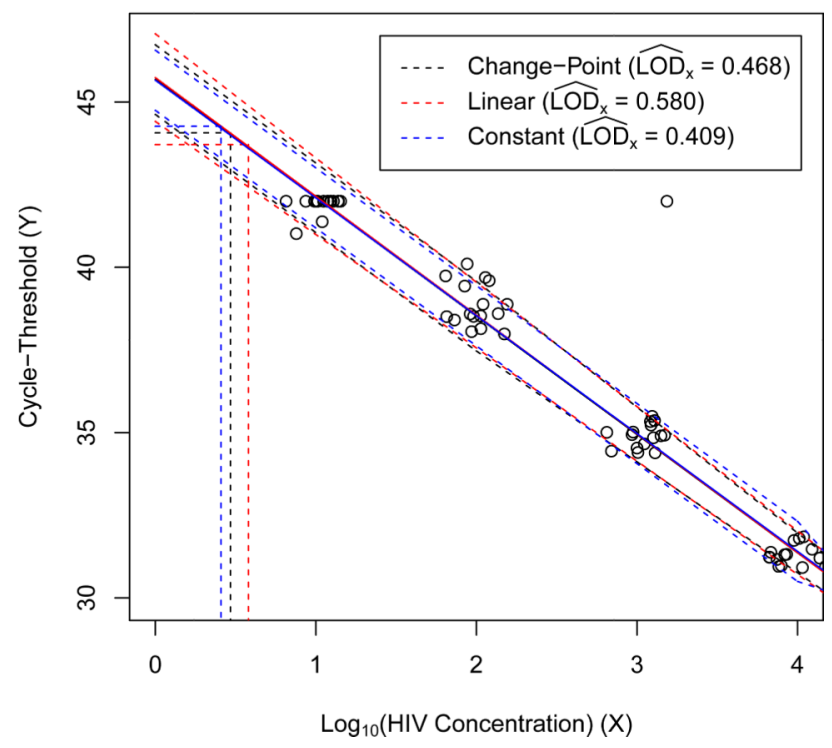

Figure 1.

Change-point model results for experiments 1 and 3 


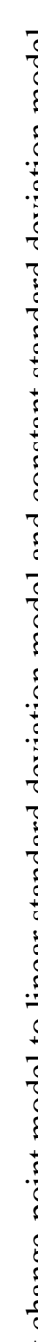

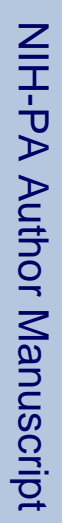

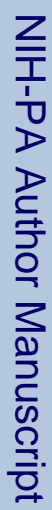

\begin{tabular}{|c|c|c|c|c|}
\hline के & 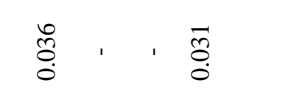 & 喜 & : & 喜， \\
\hline 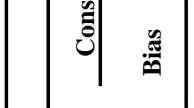 & 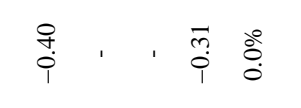 & 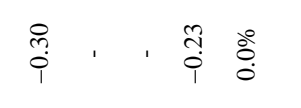 & 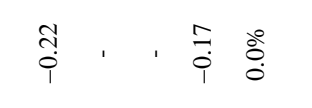 & 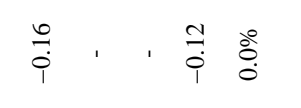 \\
\hline के & 总 & 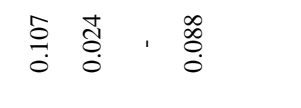 & 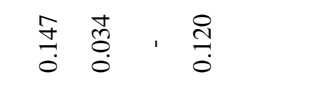 & శ్రి \\
\hline 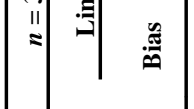 & 정 $\frac{9}{0} \cdot \frac{9}{8}$ & 我 & : & 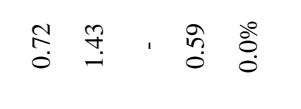 \\
\hline के & $\begin{array}{ll}8 \\
0 \\
0\end{array}$ & 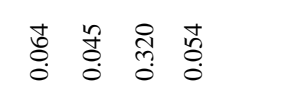 & 总高 & 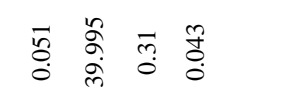 \\
\hline$\stackrel{\mathscr{m}}{\ddot{m}}$ & 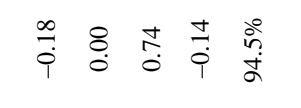 & 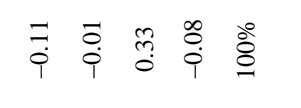 & 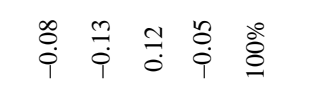 & $\stackrel{n}{0}$ \\
\hline के & 营， 嘉 & 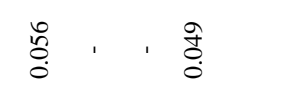 & 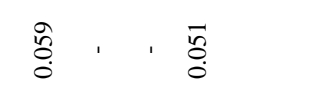 & 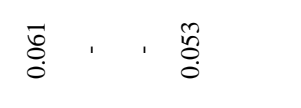 \\
\hline 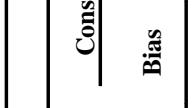 & 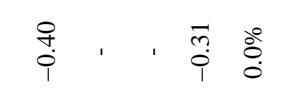 & 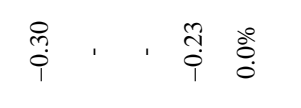 & $\underset{i}{\pi}, \quad, \frac{0}{i}$ & $\stackrel{0}{i}, \quad \overline{\bar{i}}$ \\
\hline के & 잉 & $\frac{\bar{n}}{0} \frac{0}{0}$ & 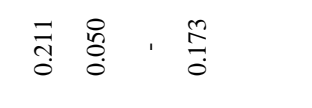 & 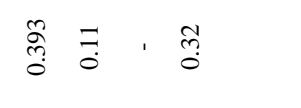 \\
\hline$\frac{2}{\underline{m}}$ & 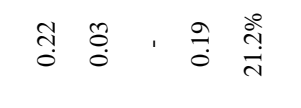 & 串 & శ్రి & $\stackrel{g}{g} \cdot{ }_{0}^{\infty}$ \\
\hline$\theta$ & 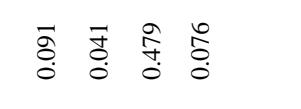 & 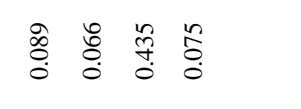 & 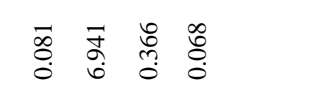 & 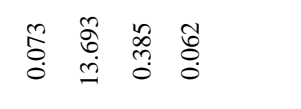 \\
\hline 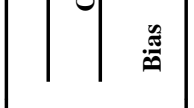 & 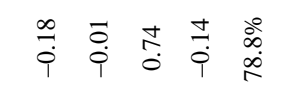 & $\overline{\bar{i}} \overline{\bar{i}} \bar{i}$ & 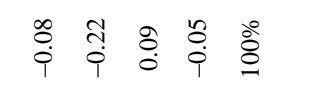 & 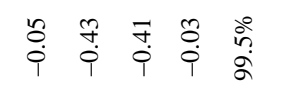 \\
\hline के & $\stackrel{8}{\circ}$ & 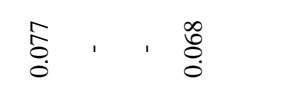 & 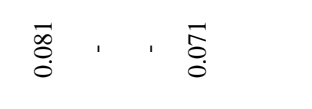 & 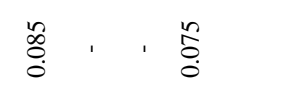 \\
\hline$\stackrel{\mathscr{m}}{\ddot{m}}$ & 旁, , 商高 & $\overrightarrow{\bar{i}}, \cdot \frac{\pi}{i}$ & $\frac{\pi}{i} \cdot \frac{n}{i} \stackrel{n}{0}$ & $\overline{\bar{i}} \cdot \overline{\bar{i}}$ \\
\hline के & $\frac{5}{0} \frac{0}{0}$ & 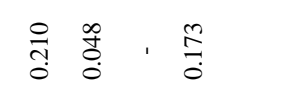 & 总 & 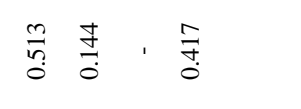 \\
\hline 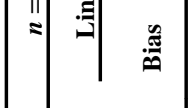 & 정 & 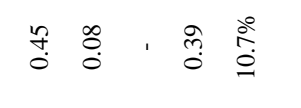 & 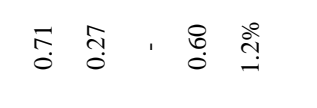 & 总表, 品 \\
\hline$\cdots$ & $\frac{\pi}{3} \frac{0}{0}: \frac{0}{0}$ & 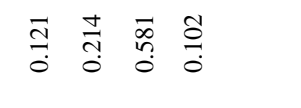 & 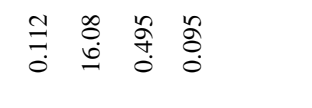 & 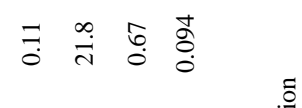 \\
\hline 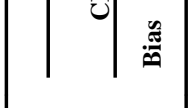 & 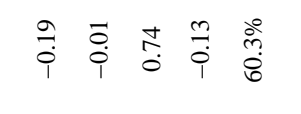 & 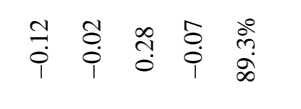 & $\begin{array}{lll}0 & 0 \\
i\end{array}$ & 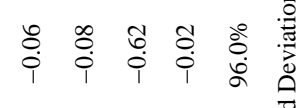 \\
\hline$\stackrel{2}{Z}$ & $=\stackrel{n}{a}=\stackrel{8}{a}$ & 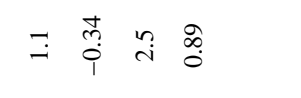 & 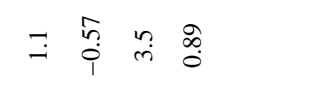 & 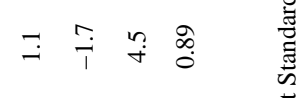 \\
\hline
\end{tabular}

Stat Med. Author manuscript; available in PMC 2014 December 10. 


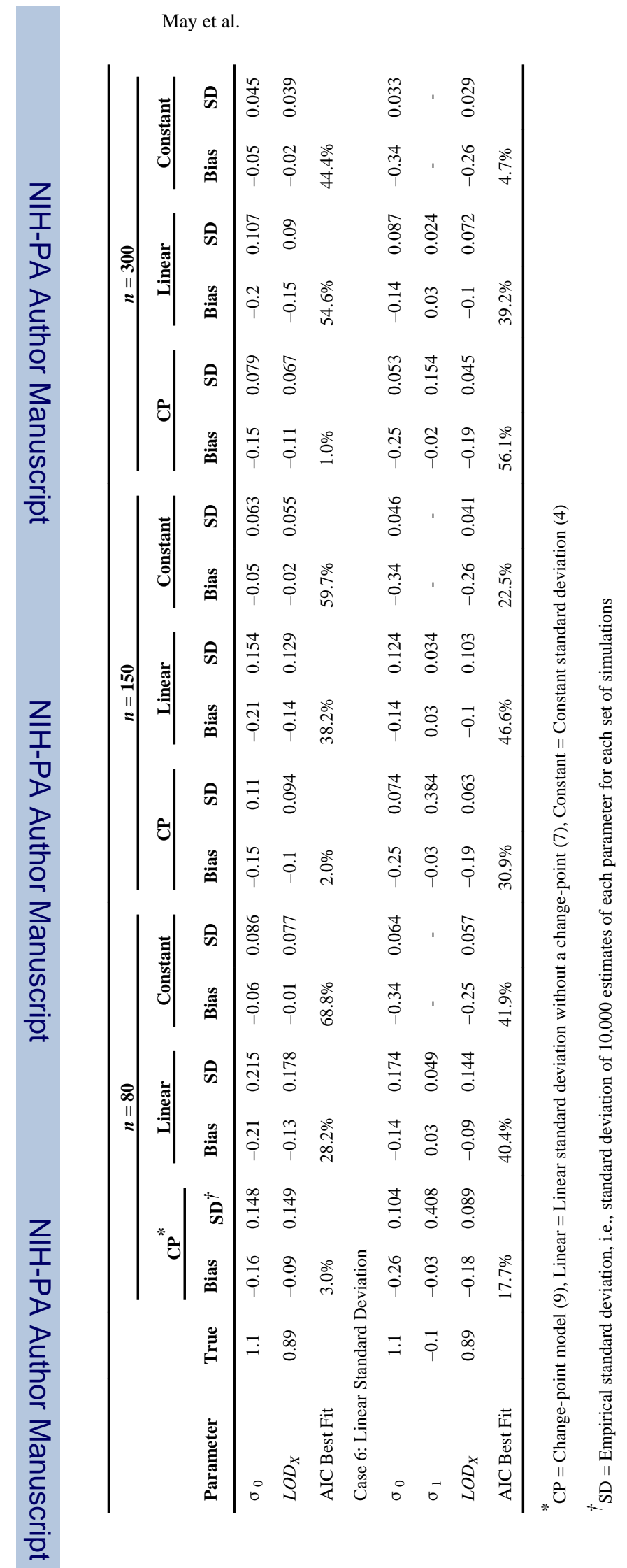


Table 2

Parameter estimates and bootstrap standard errors for HIV study, using regression models and mixed models

\begin{tabular}{|c|c|c|c|c|c|c|c|c|}
\hline \multirow[b]{2}{*}{ Experiment } & \multirow[b]{2}{*}{$\hat{\beta}_{0}$} & \multicolumn{6}{|c|}{ Regression Model } & \multirow[b]{2}{*}{ AIC } \\
\hline & & $\hat{\beta}_{1}$ & $\hat{\sigma}_{0}$ & $\hat{\sigma}_{1}$ & $\lambda$ & $\widehat{L O D}_{X}$ & ${ }_{10}^{\widehat{L O D}} X$ & \\
\hline \multicolumn{9}{|c|}{ Change-Point Model } \\
\hline 1 & $43.19(0.26)$ & $-3.661(0.077)$ & $1.147(0.106)$ & $-0.278(0.038)$ & $1.000(0.00)$ & $0.959(0.083)$ & $9.10(1.70)$ & 181.68 \\
\hline 2 & $45.20(0.29)$ & $-4.104(0.079)$ & $1.612(0.181)$ & $-0.452(0.067)$ & $1.000(0.09)$ & $1.195(0.131)$ & $15.68(4.94)$ & 235.64 \\
\hline 3 & $45.68(0.16)$ & $-3.579(0.049)$ & $0.537(0.092)$ & $-0.115(0.051)$ & $2.000(0.22)$ & $0.468(0.077)$ & $2.94(0.50)$ & 85.41 \\
\hline 4 & $45.35(0.17)$ & $-3.472(0.047)$ & $0.747(0.109)$ & $-0.255(0.042)$ & $1.699(0.32)$ & $0.661(0.093)$ & $4.58(1.04)$ & 118.93 \\
\hline 5 & $42.42(0.22)$ & $-3.395(0.065)$ & $1.186(0.100)$ & $-0.262(0.037)$ & $1.000(0.06)$ & $1.063(0.084)$ & $11.57(2.22)$ & 291.30 \\
\hline 6 & $43.17(0.22)$ & $-3.684(0.064)$ & $1.262(0.089)$ & $-0.310(0.033)$ & $1.000(0.00)$ & $1.041(0.074)$ & $11.00(1.86)$ & 286.98 \\
\hline \multicolumn{9}{|c|}{ Linear Standard Deviation Model } \\
\hline 1 & $43.19(0.25)$ & $-3.661(0.076)$ & $1.425(0.138)$ & $-0.278(0.037)$ & - & $1.183(0.108)$ & $15.23(3.74)$ & 179.68 \\
\hline 2 & $45.20(0.30)$ & $-4.104(0.081)$ & $2.062(0.243)$ & $-0.452(0.066)$ & - & $1.522(0.173)$ & $33.25(14.21)$ & 233.64 \\
\hline 3 & $45.74(0.18)$ & $-3.593(0.061)$ & $0.676(0.230)$ & $-0.085(0.078)$ & - & $0.580(0.181)$ & $3.80(1.42)$ & 86.88 \\
\hline 4 & $45.43(0.18)$ & $-3.492(0.048)$ & $1.118(0.152)$ & $-0.239(0.038)$ & - & $0.971(0.126)$ & $9.36(2.82)$ & 118.10 \\
\hline 5 & $42.42(0.21)$ & $-3.395(0.065)$ & $1.448(0.143)$ & $-0.262(0.039)$ & - & $1.292(0.120)$ & $19.60(5.50)$ & 289.30 \\
\hline 6 & $43.17(0.22)$ & $-3.684(0.064)$ & $1.572(0.118)$ & $-0.310(0.033)$ & - & $1.291(0.097)$ & $19.55(4.46)$ & 284.98 \\
\hline \multicolumn{9}{|c|}{ Constant Standard Deviation Model } \\
\hline 1 & $43.26(0.37)$ & $-3.688(0.118)$ & $0.920(0.096)$ & - & - & $0.776(0.074)$ & $5.96(0.99)$ & 219.63 \\
\hline 2 & $44.95(0.39)$ & $-4.004(0.118)$ & $1.199(0.136)$ & - & - & $0.927(0.109)$ & $8.46(2.18)$ & 294.05 \\
\hline 3 & $45.66(0.14)$ & $-3.563(0.043)$ & $0.464(0.060)$ & - & - & $0.409(0.052)$ & $2.56(0.30)$ & 88.08 \\
\hline 4 & $45.27(0.19)$ & $-3.424(0.056)$ & $0.622(0.083)$ & - & - & $0.565(0.075)$ & $3.67(0.62)$ & 156.98 \\
\hline 5 & $42.44(0.26)$ & $-3.406(0.083)$ & $0.920(0.080)$ & - & - & $0.830(0.064)$ & $6.76(0.99)$ & 326.45 \\
\hline 6 & $43.53(0.29)$ & $-3.825(0.095)$ & $0.972(0.076)$ & - & - & $0.781(0.058)$ & $6.04(0.79)$ & 339.77 \\
\hline
\end{tabular}

\begin{tabular}{|c|c|c|c|c|c|c|c|c|}
\hline \multirow[b]{2}{*}{ Experiment } & \multicolumn{8}{|c|}{ Mixed Model } \\
\hline & $\hat{\beta}_{0}$ & $\hat{\beta}_{1}$ & $\hat{\sigma}_{0}$ & $\hat{\sigma}_{1}$ & $\lambda_{i}$ & $\widehat{L O D}_{X}$ & ${ }_{10 L O D}$ & AIC \\
\hline \multicolumn{9}{|c|}{ Change-Point Model } \\
\hline All & 44.37 & -3.698 & 1.342 & -0.313 & $*$ & 1.190 & 15.49 & 2955.9 \\
\hline \multicolumn{9}{|c|}{ Linear Standard Deviation Model } \\
\hline All & 44.38 & -3.703 & 1.459 & -0.250 & - & 1.273 & 18.75 & 2994.0 \\
\hline \multicolumn{9}{|c|}{ Constant Standard Deviation Model } \\
\hline All & 44.43 & -3.725 & 0.973 & - & - & 0.910 & 8.13 & 3240.4 \\
\hline
\end{tabular}

